\title{
Personaje autónomo y yo narrativo. Una lectura de Bajo la red, de Iris Murdoch, en el marco de la perspectiva fenomenológica de Paul Ricoeur ${ }^{1}$
}

Autonomous character And narrative Self. A reading of Under the Net, by Iris Murdoch, Within the framework of Paul Ricoeur's phenomenological perspective

Andrea Coghi*

* Benemérita Universidad Autónoma de Puebla, México

Correo-e: acoghitec@gmail.com (D)https://orcid.org/-0003-0000 0725-1398 Recibido: 18 de junio de 2019 Aprobado: 22 de mayo de 2020
Resumen: Se analizan las dimensiones del yo narrativo —en cuanto tipología especial distinta y al mismo tiempo relacionada con el yo ontológico de la filosofía y el yo de la reflexión ética, descrito por Paul Ricoeur en Sí mismo como otro — en la identidad y posible autonomía de los personajes literarios. Se aplican las consideraciones de su estudio como método de análisis en los ejemplos provistos por una novela de la literatura inglesa del siglo XX, Under the Net [Bajo la red] (1954), obra de Iris Murdoch, una reconocida autora tanto de literatura como de filosofía, particularmente sensible a los temas de autonomía del individuo en la ficción en cuanto reflejo de la naturaleza humana.

Palabras clave: novela; obra literaria representativa; literatura contemporánea; literatura europea; análisis literario; filosofía; ontología; ética

Abstract: The dimensions of the narrative I - as a special typology different but related at once to the ontological I of philosophy and the I of ethical reflection described by Paul Ricoeur in Oneself as another - are analyzed in the identity and possible autonomy of literary characters. The considerations of his study are applied as an analysis method in the instances provided by a XX-century literary English novel, Under the net (1954) by Iris Murdoch; a renowned author of literature and philosophy, particularly attentive to the autonomy of individuals in fiction as a reflection of human nature.

Keywords: novels; representative literary works; contemporary literature; European literature; literary analysis; philosophy; ontology; ethics

1 Dedicado a la memoria del Dr. Enrique Pérez Castillo, profesor y guía, cuyas enseñanzas fueron imprescindibles para la elaboración del presente trabajo. 


\section{INTRODUCCIÓN}

La línea que separa el estudio filosófico de la composición literaria, la posibilidad de presencia de uno al interior del otro - en ambas direcciones-, y la coexistencia del filósofo y del autor de literatura en la misma persona, en particular en novelas, constituyen un grupo temático de reflexión que ha apasionado a más de un estudioso en la historia de ambas formas de escritura. En una entrevista de 1965 para la emisión Modern Novelists de la BBC, el crítico Frank Kermode presentó a la irlandesa Iris Murdoch con palabras que subrayaban la relación entre las dos disciplinas que practicaba, ficción y filosofía: "ambas tratan de la naturaleza humana, pero con herramientas diferentes, y si una persona hace ambas actividades, éstas están destinadas a ser afectadas de maneras interesantes" [la traducción es mía] (Murdoch, 1965). Incluso admitiendo una conexión y un mutuo soporte entre las dos áreas, la autora afirma en la misma entrevista que, como filósofa moral, "escribir filosofía es una cosa bien distinta de escribir ficción, es un trabajo diferente que apunta a resultados diferentes [...] pero la materia de estudio es la misma, o sea, la naturaleza humana en acción" [la traducción es mía] (Murdoch, 1965).

Si por un lado podemos imaginar una insuperable distinción entre los seres humanos reales y los personajes de ficción, por otro, vale la pena recordar las palabras con las cuales la misma escritora cierra la entrevista: "Amo realmente a mis personajes, la dificultad es que uno tiene que ser capaz de dejarlos libres, de hacerlos independientes" [la traducción es mía] (Murdoch, 1965). En congruencia con su intención, en la primera de sus novelas es posible percibir una autonomía de movimiento, cierta individualidad en las acciones, sentimientos y pensamientos de la que sus personajes gozan y que no sólo hace de la lectura una experiencia particularmente viva y por eso entretenida, sino que permite al lector reflexionar sobre los éxitos, fracasos y descubrimientos de los individuos que pueblan sus páginas, sin razones para dudar de la validez de las reflexiones en cuanto aplicadas a creaciones de la mente de otra persona. Sin duda, los personajes de estas obras constituyen un buen ejemplo de yo narrativo autónomo en cuanto se trata de seres únicos y coherentes, merecedores de las mismas observaciones que un yo real.

Con respecto a la construcción del argumento, se puede considerar la visión apenas citada de Murdoch sobre la independencia de los personajes como una reelaboración de una notatesis de Henry James (1934: 42-48) en la que el novelista considera que el desarrollo del carácter merece igual consideración, si no superior, que el de la trama. ${ }^{2}$ Aún más, específicamente la construcción de un yo narrativo autónomo se puede entender como un recurso específico de la configuración narrativa. El objetivo del presente estudio es identificar algunos caracteres subyacentes a la definición del individuo - como persona y sujeto de la acción narrativa-, analizando sus dimensiones ontológicas, para luego poder observar la manera en que, en una obra literaria, los cambios en la percepción de sí de los personajes afectan y enriquecen la trama.

Para la definición del yo narrativo y para una serie de consideraciones sobre los puntos de contacto entre los individuos reales y sus contrapartes artísticas - los personajes literarioses preciso considerar una parte de la obra del filósofo francés Paul Ricoeur. En ésta podemos encontrar un análisis fenomenológico de varios aspectos del yo — desde una perspectiva semántica, pragmática y ética-, incluyendo la posibilidad de que su configuración como individuo literario autónomo (en particular en las lecturas de grandes filósofos y críticos, entre los cuales se encuentra el mismo Frank Kermode) pueda contribuir a identificar tipologías, papeles y

2 Se insertan en la misma línea las observaciones de Kermode (1978: 81), quien afirma que para desarrollar un carácter se debe narrar más, y para desarrollar una trama hay que enriquecer un carácter. 
responsabilidades, así como ser considerado por varias ramas de la filosofía. Tomando en cuenta el estudio de Ricoeur, el yo narrativo no sólo no está irremediablemente separado de otras dimensiones del ser humano sino que, en muchas de las reflexiones que surgen sobre esa figura ficcional, ayuda a la definición filosófica y permite meditaciones sobre las implicaciones éticas del yo extranarrativo. Particularmente interesante en la visión del filósofo francés es la consideración del hecho de que "no existe narración éticamente neutral. La literatura es un vasto laboratorio en el que experimentamos con estimaciones, evaluaciones y juicios de aprobación y de condena, a través de los cuales la narración sirve como propedéutica a la ética” [la traducción es mía] (Ricoeur, 1990: 115).

Si por un lado Ricoeur y los referentes citados en su obra logran utilizar la narración a fin de llegar a puntualizaciones filosóficas — esenciales, ontológicas o éticas-, por otro, emerge de su trabajo una identidad literaria enriquecida, más completa en su dimensión de autonomía, en su distinción entre yo ipse y yo idem, consciente de sus implicaciones éticas en los textos. En cierto sentido, es la misma exhaustividad de esta sistematización de la identidad narrativa, a lo largo de dos obras fundamentales de su producción (Tiempo y narración, de 1985, y Oneself as another [Sí mismo como otro], de 1990), la que invita a su empleo como herramienta analítica literaria. De forma análoga, para encontrar el mismo doble filo (ficción-filosofía) en un objeto de estudio, se encuentra un ejemplo privilegiado, también a partir de las afirmaciones ya presentadas, en la obra de la filósofa y escritora Iris Murdoch. Por ello, se analiza su primera novela, Bajo la red [Under the net] (1954), para poner a prueba la configuración ricoeuriana del yo narrativo como herramienta de análisis y reflexión filosófica. Con base en la intención de su autora de desarrollar personajes con un elevado nivel de autonomía y de simular las dimensiones del yo extranarrativo al interior del texto, se intenta explorar tanto los significados y el potencial analítico de las definiciones de Paul Ricoeur como los alcances narrativos de la construcción voluntaria de un sujeto literario autónomo, junto con la posible expansión de su idea de configuración -incluyendo la preconfiguración y la reconfiguración, que el teórico llama mimesis I y mimesis III (1985: 113-141)—, en forma de sujeto de la acción y de la imputación moral en personajes de ficción.

\section{LAS DIMENSIONES DEL YO EN LA REFLEXIÓN de Paul Ricoeur}

En su notable obra Oneself as another (1990), Paul Ricoeur desarrolla un atento análisis del tema de la subjetividad en la filosofía occidental mediante el método de la hermenéutica fenomenológica. La compleja búsqueda para la recuperación de una dimensión del sujeto en su genuina realidad existencial pone a prueba el pensamiento de un gran número de autores anteriores - y algunos contemporáneos-, a partir de las críticas al yo exaltado, metafísico, de Descartes (Ricoeur 1990: 4-11), y a su visión contrapuesta, el yo quebrantado de Nietzsche (Ricoeur 1990: 20-16). Un importante antecedente de esta reflexión se encuentra en Tiempo y narración, estudio en el que Ricoeur ya había considerado el tema de la persona en su dimensión de identidad narrativa:

El frágil vástago, fruto de la unión de la historia y de la ficción, es la asignación a un individuo o a una comunidad de una identidad específica que podemos llamar su identidad narrativa. El término 'Identidad' es tomado aquí en el sentido de una categoría de la práctica. Decir la identidad de un individuo o de una comunidad es responder a la pregunta: iquién ha hecho esta acción?, ¿quién es su agente, su autor? (1985: 997). 
Tiempo y narración ofrece un primer esbozo de las perspectivas del autor respecto al tema, introduce los conceptos de idem (1985: 971) e ipse (1985: 998), y establece un primer punto de vista sobre la inestabilidad de la identidad (1985: 1001). Sin embargo, debido a los objetivos de esta obra, centrada en las posibles soluciones de las aporías del tiempo cosmológico y el tiempo fenomenológico, los aspectos de las dimensiones del yo y la consciencia de sí del personaje literario se mantienen al margen del discurso y no se profundiza en la relación entre los acontecimientos de la trama y su percepción del personaje en cuanto entidad autónoma y sujeto presente dentro de su entorno. Es más, si la identidad narrativa es, como afirma Ricoeur en las conclusiones (1985: 994, 1000, 1036), la respuesta principal para las aporías del tiempo objeto de su estudio, en una dimensión estrictamente literaria el desarrollo del personaje en la preconfiguración narrativa - lo que en la primera parte define como mimesis I (1985: 116-177)-, y en sus acciones en la configuración de la historia — es decir, mimesis II (1985: 131-133)—, entonces, cierta forma de independencia puede devolver a esta figura la centralidad que Aristóteles le negaba, en favor de la trama (1985: 90). Esto, lejos de ser un principio absoluto, constituye por lo menos una posibilidad, como se observa en el presente estudio, en ciertos modos narrativos como la novela moderna - en contraste con el ejemplo típico de Aristóteles, la tragedia griega-, en particular, en el caso de una obra construida alrededor del concepto de personaje autónomo.

Para desarrollar este tipo de precisiones, vale la pena que observemos la manera en que el análisis de los aspectos narrativos del yo en Oneself as another (1990: 113: 168) se encuentra entre los dos extremos señalados, representados mediante las posturas de Descartes y Nietzsche. Se trata, por lo tanto, de una redefinición ontológica de la identidad del individuo a partir de la separación de un yo ipse y un yo idem (1990:
88-168) y del establecimiento de una relación de estas dos dimensiones con la otredad que los demás individuos le atribuyen y que, en varios casos, sirve para definirlos. Para el estudio del tema, Ricoeur descompone su análisis en tres partes: en la primera (estudios del 1 al 4), se cruzan semántica y pragmática con la filosofía del lenguaje y la filosofía de la acción. En la segunda, idem e ipse encuentran definiciones específicas gracias al estudio de su aspecto temporal mediante la identidad narrativa (estudios 5 y 6). En la tercera, el yo es sujeto a la determinación de sus dimensiones éticas y morales en relación con las categorías de lo bueno y lo obligatorio, y la aclaración del papel de la otredad en la definición del sujeto (estudios 7, 8 y 9).

Al final de este breve resumen, y buscando considerar el alcance general de la obra de Ricoeur, parece necesario aislar, para los fines del presente artículo, las consideraciones del quinto y sexto estudios (1990: 113-168). Estos capítulos desarrollan, respectivamente, un análisis — con diferencias y analogías - entre la dimensión personal y la narrativa del concepto de identidad, y un atento desarrollo del yo ipse en el contexto de la identidad narrativa misma. Cabe recordar, antes de explorar los resultados más importantes de ambos estudios, que a partir de estas dos partes del recorrido, el filósofo francés puede identificar las peculiaridades del aspecto más significativo del yo y concentrarse, sucesivamente, en los aspectos que implican el sujeto no puramente narrativo en su dimensión social y su interacción con todas las formas -individuales, colectivas, activas y pasivas - de la otredad.

A partir de las definiciones de mismidad —es decir, el yo reconocido por los demás en cuanto extremadamente parecido a la imagen que tienen en su mente, por lo tanto, inmutable - y de ipseidad - el yo idéntico como propio y opuesto al otro, no por simple apariencia sino por su naturaleza de extraño a cualquier identidad distinta-, Ricoeur nos recuerda que en sus estudios 
anteriores aún no lograba encontrar una dimensión correcta y sin ambigüedades por la falta de consideración del eje temporal (1990: 113). El contexto de la historia de una persona, contada y recordada en la narración, es tratado en el quinto estudio como 'tiempo en conexión con la vida' (1990: 115). En este marco, el primer problema, central en toda la reflexión del texto, es la definición de la identidad personal y del papel que la ipseidad y la mismidad desarrollan en la acción contada. La narración permite superar los límites temporales de toda la reflexión, pero "a primera vista, de hecho, la cuestión de la permanencia del tiempo está conectada exclusivamente con la identidad 'misma' (idem)". Los dos criterios propuestos, identidad numérica - relacionada con la cantidad de apariciones de la misma cosa o persona llamada con cierto nombre- y cualitativa -el extremo parecido entre dos cosas o personas, al punto de que éstas son intercambiables-, categorías propuestas por Kant, se encuentran presentados junto con sus límites (Ricoeur 1990: 116). No es difícil pensar que la identificación de una persona con sí misma puede dar espacio a ambigüedades, frente a criterios considerados alrededor de su idea de parecido a la imagen mental en los demás, lo cual pondría en conflicto la percepción de estos (como en los casos de un crimen donde hay un testigo ocular) al tener la necesidad de identificar al sujeto real: lo anterior, válido ya a partir de casos con cantidades de tiempo mínimas entre los dos momentos de reconocimiento, encuentra mayor justificación al aumentar el lapso. Por esta razón, los dos criterios propuestos son integrados por un tercero y nuevo, más compatible con la dimensión temporal: la continuidad ininterrumpida, que permite, a lo largo de la existencia de un objeto, persona o animal, aceptar la continuidad de su identidad a pesar de los cambios que, poco a poco, modifican su aspecto exterior, incluso de forma radical. De esta manera, el sistema combinatorio de los criterios tiene en cuenta el carácter relacional de la identidad con sus factores externos, y por esto se puede decir que, en particular en su aspecto idem, encuentra y supera el problema de la permanencia en el tiempo mediante su invariancia relacional (Ricoeur, 1990: 118).

Otro paso fundamental de la investigación es la búsqueda, para la superación de la cuestión del tiempo, de una definición de identidad que no se remita sólo a la sustancia, sino que tome en cuenta la ipseidad del sujeto -o bien, en palabras de Ricoeur, "una forma de permanencia en el tiempo que sea una respuesta a la pregunta ‘quién soy yo?’-(1990: 118). Su propuesta se desarrolla alrededor de "dos expresiones que son descriptivas y emblemáticas al mismo tiempo: el carácter y el mantener la palabra de uno mismo".

"Por carácter - escribe Ricoeur- entiendo el conjunto de tractos distintivos que permiten la reidentificación de un individuo humano en cuanto uno mismo" (1990: 119). Las primeras observaciones del autor hacen referencia a un estudio suyo anterior (1966), donde subraya la involuntariedad del carácter mismo, aunque éste permita el acceso a las facultades voluntarias de los valores y el uso de los poderes de uno. Pero es en el tratamiento del tema de la dimensión temporal donde se expresa la parte más relevante de su definición: la evolución del carácter de un individuo - de una manera no disímil del carácter de una comunidad- se define como identificación adquirida, es decir, es el producto de una serie de rechazos, aceptaciones y asimilaciones de hábitos entendidos como valores, normas, ideales, modelos y héroes. Si por un lado esto va delineando un perfil dinámico del carácter y una constante dialéctica cíclica entre innovación y sedimentación de los hábitos, por el otro, subraya por primera vez un tipo distinto de dialéctica entre la otredad de los hábitos mismos — que tendrá particular importancia en la investigación de las dimensiones éticas del yo, en la tercera parte de la obra-y su internalización en el carácter del individuo (Ricoeur, 1990: 121-122). 
En esta dimensión, idem e ipse se entrelazan íntimamente y tienden a fundirse en una sola versión de la identidad.

El otro modelo de permanencia de un individuo en el tiempo es descrito como el acto voluntario de autoconstancia, que se expresa en el mantenimiento de la propia palabra. El acto voluntario de tener fe en la palabra dada, dentro del marco de las posibilidades de cada individuo, implica, en el acto de la promesa, un intento de negación del cambio y, en el cumplimiento de la misma, ${ }^{3}$ el desarrollo de las funciones de salvaguarda de la institución del lenguaje, de los valores éticos de los dos sujetos -emisor y receptor-, y de una constancia del carácter en el tiempo que se opone al dinamismo de la definición anterior, que tanto lo asimilaba a las cuestiones del yo mismo (Ricoeur, 1990: 123-124).

Una vez descritos ambos conceptos, Ricoeur recuerda cómo la cuestión del carácter define mismidad e ipseidad sobrepuestas una a la otra, mientras el mantener la palabra de uno mismo separa marcadamente los dos niveles de permanencia de ambas dimensiones identitarias (1990: 118). A partir del carácter inevitable de la copresencia de estas dimensiones en sus diferencias, se abre lo que el teórico llama "un intervalo de sentido" (Ricoeur, 1990: 124): para una superación de esta polarización que dé razón a su teoría, el autor propone el estudio de la identidad narrativa en su contexto temporal y en la interacción entre estructura de la narración y acciones de sus personajes.

En el sexto estudio, se analiza la identidad del personaje literario desde dos puntos de vista: primero, su constante interacción con la configuración del tiempo del texto -0 en palabras del autor, del entramado - nos permite entender mejor el nuevo papel del yo ipse; segundo, éste (en su renovada definición propia, pero siempre

3 Ricoeur reconoce -implícitamente en Oneself as another (1990: 183), y de forma explícita en Tiempo y narración (1985: 1001) — ser deudor de las observaciones de Levinas. indisolublemente en dialéctica con su yo mismo) es analizado en las funciones de los personajes literarios según los métodos de la teoría narrativa. Un concepto fundamental del capítulo es, entonces, en la interacción con la trama, la 'competición dinámica entre la demanda de concordancia y la concesión de discordancias' (Ricoeur, 1990: 141), es decir, el orden de la historia necesita de la tensión, de los desórdenes provocados por los cambios en los destinos para que la estructura narrativa tenga su desarrollo. Para las finalidades filosóficas de su estudio, Ricoeur nos explica cómo el mismo esquema opera no sólo a nivel de la trama sino en la configuración de los personajes, o sea, en la forma de una dialéctica entre el retrato profundo de estos últimos y los cambios necesarios en su conducta, acciones y maneras de pensar, demostrando las evoluciones provocadas por la historia (o necesarias para suscitar su desenlace).

Cabe recordar, en primer lugar, el soporte de algunos esquemas de análisis de otros estudiosos que enriquecen la reflexión de Ricoeur, en particular, el concepto de Claude Bremond de una trama como distribución de recompensas y puniciones, en la que los personajes alcanzan una autonomía y se hacen iniciadores de sus propias acciones en el momento que pasan de ser receptores a ser distribuidores de éstas (Ricoeur, 1990: 145). Además, en cuanto al tema de las evoluciones, en un pasaje significativo de las conclusiones de Tiempo y narración se recuerda la posibilidad de un doble dinamismo, del personaje y de la narración de sí que, si bien está referido a la identidad narrativa en textos diferentes, integra bien las reflexiones sobre la interacción entre el quién y el qué en la trama: "[L]a identidad narrativa no es una identidad estable y sin fisura: y así como se pueden componer diversas tramas a propósito de los mismos sucesos [...], igualmente siempre es posible urdir sobre su propia vida tramas diferentes, incluso opuestas" (Ricoeur, 1985: 1000). 
Gracias al nuevo papel del personaje en su identidad autónoma, se puede encontrar en la segunda parte del capítulo una serie de paralelismos entre la narración y la vida real y, a pesar de las diferencias constitutivas entre ambas dimensiones - entre las más destacables, la que Ricoeur, citando a Frank Kermode, llama 'el sentido de un final'-, la posibilidad de la historia de constituir un laboratorio de comprensión de tres niveles de la existencia extranarrativa. El primero está compuesto por la operación definida por la expresión diltheyana 'la conectividad de la vida', que MacIntyre llama 'la unidad narrativa de la vida' (Ricoeur, 1990: 157). En ésta, un plan general - susceptible de infinitas revisionesda sentido a la transformación de las acciones prácticas en una vida completa (para usar palabras de Aristóteles, las categorías de praxis y bios narradas mediante la mimesis literaria). En un segundo nivel (Ricoeur, 1990: 158), hallamos la narración de la vida en su sentido de buena vida, que constituye la piedra angular de la ética. En un último nivel, se presenta la posibilidad de la literatura de exorcizar los miedos a lo desconocido y a la muerte con sus formas de encerrar la desolación humana en las categorías de ordenamiento superior y el consuelo de la katharsis.

Más allá de su importante alcance filosófico, estas consideraciones sobre la relación narración-identidad personal, y su gran número de indicaciones sobre aspectos de la literatura, pueden encontrar una posible relevancia, como ya se dijo, que justifica el uso de las categorías definidas a manera de método para el análisis de la preconfiguración y configuración ficcional basada en el personaje, sobre todo en la dimensión mimética del relato novelesco. El experimento del apartado siguiente intenta poner en práctica una identificación de los elementos ricoeurianos señalados hasta ahora, observando el desarrollo y la puesta en acción de personajes 'independientes' en una obra de Iris Murdoch.
LA AUTONOMÍA DEL PERSONAJE Y LA TRAMA

Bajo la red, la primera obra de ficción de Iris Murdoch, publicada en 1954, encontró inmediatamente una buena apreciación de la crítica y el público — de forma mayor que cualquiera de sus siguientes obras-, y en 1998 fue considerada como una de las cien mejores novelas de lengua inglesa del siglo XX. En el momento de su publicación, la autora era una afamada profesora en Oxford y había escrito algunos ensayos de filosofía, en particular, la primera monografía publicada sobre Jean-Paul Sartre. Bajo la red es una novela en la que elementos de autoconciencia del protagonista y un evidente desarrollo del personaje integran la aparente naturaleza cuasipicaresca de la narración episódica. ${ }^{4}$ En el texto se entrelazan escenas grotescas, comparaciones entre varias posturas filosóficas, profundas consideraciones sobre el amor, y se evidencia cierto esfuerzo del ser humano para comprender el sentido de su existencia. La obra y la doble actividad de escritora y filósofa de Murdoch despertaron desde el inicio la curiosidad de los estudiosos de ambas ciencias humanas y varios de ellos se cuestionaron sobre la posibilidad de mantener los dos tipos de producción sin exceder la interferencia mutua y la conmixtión de géneros. En una entrevista concedida a Bryan Magee (Murdoch, 1978), la autora insistió largamente en que, aun coincidiendo el objeto de estudio, los intentos de los dos tipos de texto son radicalmente diferentes. A consideración de los puntos comunes, afirmó que se encuentran ideas filosóficas que a veces son recogidas por escritores, por ejemplo, "el hecho de que el yo-mismo no es un tipo de objeto unitario, el yo-mismo es fragmentario" [la traducción es mía] (Murdoch, 1978).

4 Una vez más, para la relación entre los episodios y el poder unificador de la trama se puede hacer referencia a las observaciones de Ricoeur, en gran parte tomadas a préstamo de Aristóteles, en Tiempo y narración (1985: 98, 406). 
En ocasión de la entrevista a la autora, ya citada en la introducción al presente trabajo, el crítico Frank Kermode recuerda que "los cuentos viven separadamente de quien los cuenta" [la traducción es mía] (Murdoch, 1965), y evoca el concepto de autonomía, repitiendo las palabras de la escritora - "La señora Murdoch dice que crear personajes libres en una novela da al escritor una sensación que es cercana al amor por sus personajes" [la traducción es mía]-, pero también pone en duda que la construcción de tramas complejas y articuladas no pueda limitar la libertad de acción de estos últimos (Murdoch, 1965).

El protagonista de la novela, Jake Donaghue, es un traductor y escritor con escaso éxito, que comienza una serie de aventuras a partir de situaciones fuera del control de su voluntad, mismas que subrayan el carácter de parasitismo de su existencia reciente. Una vez echado a la calle por su exnovia, debido a la necesidad de hallar un nuevo lugar donde vivir, el personaje se mueve constantemente por Londres y París en una sucesión de encuentros, entre nuevas y viejas amistades, y va armando rápidamente planes de recuperación de una vida que parecía haber olvidado en la pasividad de los últimos años. Así, el protagonista llega a transformar la trama en una procesión de entrelazadas búsquedas: de la mujer que siempre ha amado, de un amigo perdido hace mucho tiempo, de un trabajo, de una posible seguridad económica.

El retrato que se hace de Jake y sus intentos de alcanzar un equilibrio nos permiten ver una elevada consideración de su identidad personal y de la gente a su alrededor, en particular de su amigo Finn, presentado como su sombra, un hombre "sin mucha vida interior [...] por eso [...] me sigue por donde vaya, en cuanto yo tengo una muy intensa y altamente diferenciada. De todos modos, yo cuento a Finn como un habitante de mi universo, y no puedo concebir que él tenga uno que me contenga" (9). ${ }^{5}$

5 Todas las citas pertenecientes a Bajo la red corresponden a Murdoch, 1954, por lo cual sólo se anota la página.
Durante todo el texto, aparece constantemente la vida interior del narrador-protagonista y una atención a sus percepciones en todos los niveles. La identidad de los otros personajes se define con colores dignos de caricaturas extremas, no sólo en el caso de Finn: la exnovia tiene como trabajo principal el "ser sí misma y en eso dedica un tremendo celo y sentido artístico" (10); Mrs. Tinckham es, literalmente, una fumadora serial, y no se sabe de dónde prende el primer cigarrillo de la mañana (16); Dave, "a pesar de sí mismo, es un buen profesor [de filosofía]. [...] Su gran objetivo es disuadir a los jóvenes de la filosofía”. La amada Anne es inteligente y fascinante, y parece justificar el porqué de tantos años sin contacto con Jake al definir negativamente el amor y agregar que "es persecución. Todo lo que quiero es ser dejada sola para hacer un poco de amorío por mi cuenta" (40); finalmente, su hermana Sadie, superficial actriz de éxito, parece no considerarlo, pero, "era notoriamente una mentirosa [...], yo sabía cuánto fácilmente ella era impresionada por los hombres que imaginaba ser intelectuales [como Jake] (68)".

No solamente estas personas obedecen a patrones muy claros en la mente del protagonista. Todo resulta anecdótico en la visión con la que pone en marcha la vida después de que los eventos lo obligan a moverse. La filosofía constituye rígidamente para él un conjunto de frases a efecto para desarmar el sentido de la disciplina misma - "Hegel dice que la verdad es una palabra más grande y que la cosa es más grande aún" (22); "Después de todo, la libertad es sólo una idea" (27) - , o se reduce a un sistema de citas - ' 'es en la tercer Critique [Kantiana]' Dave gritó a alguien al fondo del salón" (27)—, o a cuestionamientos que quedan suspendidos entre jóvenes universitarios - “¿cómo sabes cuál es tu verdadero yo-mismo? alguien preguntó" (28)-. Igualmente, los recuerdos de su amigo Hugo, y del libro que éste le inspiró casi plagiando sus pensamientos, están congelados en el pasado, y al recordarlo, Jake no duda de la fidelidad de su 
cuento y de lo que imagina ser el rencor de su amigo (60-67). Hasta la afirmación "Todo lo que está al Oeste de Earls Court es contingente [...]. Yo odio la contingencia. Quiero que todo en mi vida tenga una razón suficiente" (24), nos recuerda su rechazo por la indeterminación: en su plan a corto, medio y largo plazo existe un claro orden de intentos, objetivos y resultados esperados.

Y es exactamente sobre sí mismo que el protagonista proyecta la mayor cantidad de definiciones claras y monolíticas: a partir de su cuerpo y su carácter (21), su condición económica - "no pertenezco a una clase social que recibe dinero en situaciones de este tipo [...]. Lo que quedaba de mi honor estaba en juego" (72)—, sus hábitos, casi inmutables consideraciones morales - "yo consideraría inmoral ser íntimo con más de cuatro personas en cualquier momento" (24)-, y sus preferencias expresadas como sustancias ontológicas - "Odio la soledad, pero tengo miedo de la intimidad. La sustancia [iinmutable?] de mi vida es una conversación privada con mí mismo, que al convertirla en un diálogo sería equivalente a la autodestrucción" (31)—.

Por toda la primera mitad de la novela, la personalidad del protagonista, a pesar de las vicisitudes que guían el curso de sus acciones más allá de su voluntad, subraya su calidad centradora del discurso narrativo. La fuerte autonomía del personaje, que quiere escribir su historia - "lo que es más importante para las finalidades de este cuento es que tengo nervios destrozados. No importa cómo es que los tengo. Esa es otra historia" (21) - y la incontrolable complejidad de la trama, siguen en dos planos paralelos sin darse claras posibilidades de influencia recíproca: aunque adelanta en el lector la imagen de los objetivos que Jake tiene que conseguir a partir de su fuerza de determinación, por el momento, las adversidades construyen el entramado y nos presentan una visión del yo narrativo concentrada en la rigidez de un carácter que parece ignorar su dimensión temporal, su capacidad de cambiar, adaptarse e interactuar con las fuerzas presentes. La mismidad y la ipseidad del protagonista y el uso casi solipsístico que hace del otro en su cuento mantienen personaje e historia en una insostenible condición de separación, que tiene que encontrar un desenlace a partir del uso de la palabra para llegar a esquemas diferentes de actuación.

\section{EL PROTAGONISTA Y LA CONTINGENCIA DE LA VIDA Y DE LA PALABRA}

La posibilidad de un plan de vida distinto se insinúa a Jake y al ojo del lector mediante varias ideas y situaciones; en algunos casos, la misma reinterpretación, en un sentido más amplio, de su narración, dispone los elementos en favor de una restructuración de su conducta. La palabra clave con la que el personaje tiene que aprender a convivir es 'contingencia', a la cual le declara todo su odio al presentarla (24). La reacción despectiva de Jake sigue siendo fuerte en el momento en que visita los estudios de la casa cinematográfica de su examigo y, aunque lingüísticamente despersonalizada, introduce la necesidad de un cambio de postura: "El Bounty Belfounder Studio se sitúa en un suburbio del Sur de Londres donde la contingencia alcanza niveles de náusea" (139). Tal vez, el inicio de una apertura hacia una actitud menos determinista encuentra inspiración en la relectura de una parte del libro en el que plagiaba a su amigo, y en la que se incluye una de las citas más importantes de la novela, que también justifica su título:

\footnotetext{
¿Una teoría te ha ayudado alguna vez? Todo tipo de teorización es un vuelo. Tenemos que ser gobernados por la situación misma y esto es particular a un punto inexpresable. De hecho es algo a lo que no podemos nunca llegar suficientemente cerca, por cuanto lo intentemos, como si fuera a arrastrarse bajo la red (80).
} 
La nulidad de las teorías filosóficas prefiguran al narrador de su misma historia la necesidad de constantes adaptaciones más abiertas a la configuración que se le presenta: Jake no es capaz de entender el sentido de lo que éstas significaban, pero la constancia de la trama y de estos mensajes mueve algo en su modo de acción. La contingencia empieza a operar una transformación en sus consideraciones, desde las más íntimas - "ahora no sentí nada más que una determinación vacía" (91) - a las aparentemente ajenas - "ni Europa seguirá adelante para siempre, nada sigue para siempre" (101)—. Las palabras más profundas de la filosofía salen a la luz ahora, también a partir de citas de personajes de la política: el sindicalista de extrema izquierda cita, "Marx dijo muchas cosas profundas [...] por ejemplo, dijo que la conciencia no se encuentra en el ser, sino que el ser social es la fundación de la conciencia" (99-100).

La imagen del ser humano que se arrastra bajo la red no es tan diferente del cambio de posición, inicialmente de pie, luego acostado, de un hombre proyectado al piso por un movimiento de judo (145), y su caída en el espacio de un templo antiguo acentúa el contraste informalidad-sacralidad. Aún más icónica y paradójicamente relacionada con la decadencia de los imperios, así como citada algunas páginas antes, es la caída de una enorme reproducción cinematográfica: "La entera Roma estaba ahora horizontal y de sus ruinas una inmensa nube de polvo se estaba levantando" (150).

Un paso decisivo en la fuerza de la indeterminación de la realidad sobre la determinación de la voluntad de su artífice se da en los eventos que vive Jake en París: su rendición parece prepararse ya al momento de su llegada, cuando admite que la ciudad "permanece para mí una harmonía irresuelta” (168). A pesar de la sacralidad que le atribuye, el protagonista se topa con una serie de sorpresas a las que no sabe reaccionar: halla a su exnovia y no logra mantener la distancia que desea poner (172); se da cuenta de su interés por el dinero sólo después de haber rechazado irremediablemente una interesante propuesta económica de trabajo (180); espera con resolución un encuentro casual con su amada (185), se decepciona cuando esto no ocurre y al verla se pierde en un juego de persecución sin alcanzarla (194). La imposibilidad de guiar su vida lo hace regresar a Londres, frente a su necesidad de seguir buscando a Hugo sin saber cómo lograrlo, poner al tanto a Finn de su destino común y cuidar de un perro que, aunque en un inicio fue secuestrado, ahora se vuelve simplemente adoptado. Su identidad empieza a tener un sentido más ligado a las condiciones reales que se le ofrecen y, a partir de este momento, la trama le ofrece la oportunidad de seguir, esta vez con sus acciones y no en contra de éstas. Jake empieza a trabajar en un hospital y ahí encuentra, entre sus pacientes, a Hugo:

el sentido de mi propio destino $[. .$.$] ahora había$
regresado, y sentí que, cualquier cosa, dios
[con letra minúscula] ${ }^{6}$ tenía organizado para
mí y Hugo en cuanto profundamente conecta-
dos uno al otro, no habría dejado su trabajo
terminado (208).

Ahora que Jake está dispuesto a aceptar su compromiso como persona en un contexto rodeado de otros y que puede admitir lo que la realidad le ofrece, ésta no escapa más a su comprensión y se revela por lo que es, incluso las partes menos claras. La historia de amor que creía inevitable se transforma en una irresoluble cadena de atracción entre cuatro personas, donde cada una está enamorada de alguien que no le corresponde; esta vez, la respuesta de Hugo se le hace entendible: "lo sabes, cada quién puede amar a cada quién, o preferir uno a otro" (226). Hasta la búsqueda de la verdad y de Dios por parte de su amigo termina con una solución simplificadora:

6 Curiosamente, pocas páginas después, es presentado como sinónimo de una verdad filosófica, por lo que está escrito con mayúscula inicial. 
"Dios es una tarea, Dios es un detalle. Está todo al alcance de tus manos" (229).

Si durante algunas páginas el cambio de percepción es suficiente como para dejarlo en un estado de cierto extrañamiento - "no me sentía ni feliz ni triste, sólo algo irreal, como un hombre encerrado en un vaso" (244)—, ahora Jake está listo para considerar que las cosas no siguen un destino directamente ligado a su proyección mental. Cuando su preocupación por la desaparición de Finn se ha transformado en una condición normal, descubrimos que éste ha retomado la posesión de su vida independiente, regresando a su nativa Dublín, y hasta vemos por primera vez su verdadero nombre, que aparece mencionado al final de la carta que le escribe a un amigo en Londres (247). La trama presenta en estas últimas páginas elementos cíclicos con respeto al inicio, y el autor reconoce abiertamente regresar, con diferente actitud, a los temas con los que había empezado: "esto me dejaba con más o menos la misma cantidad de dinero a mi nombre de la que tenía cuando salí de Earls Court Road al inicio de esta historia" (250). También un sentido de aprendizaje y de nuevo comienzo resalta en sus últimas palabras: "Estaba lleno de esa fuerza que es mejor que la felicidad [...]. Era el inicio del primer día” (251).

\section{LA OTREDAD DE LA IDENTIFICACIÓN \\ Y EL JUICIO DEL AUTOR}

Mediante el aprendizaje que él mismo reconoce, la identidad del narrador deja de estar vinculada al carácter autorreferencial de sus proyecciones hacia los demás y a su imagen de sí impuesta por su mente en los ojos ajenos. Una de sus últimas consideraciones sobre la conversación reveladora con su amigo-paciente hace explícitas estas meditaciones en palabras de un tono más cercano a las de un filósofo que a las de un artista, como Jake pretende ser: "empecé a pensar en
Hugo. [...] Su propia otredad tenía que ser buscada no en sí mismo sino en mí mismo o Anna. Y esto porque él no reconoce nada de lo que ha hecho" (238). El amigo, en su escasa autoconsideración, no es ya una figura de referencia sino una otredad más, así como el mismo Jake puede serlo para Hugo. La dimensión de apertura hacia el mundo, que se expresa en las sorpresas y la felicidad de las últimas páginas, revela toda la vitalidad de un hombre que ha recuperado su correcta dimensión, la conciencia de la fragmentariedad de su propio ser en tanto percepción de sí mismo, así como la inevitable dimensión externa en el otro que lo configura como un alguien que necesita reflejarse en los ojos de los demás. Ahora se encuentra más dispuesto a una dialéctica honesta con su otredad, representada no sólo en las personas sino en los eventos, ese destino con el que puede enfrentarse sin excesivas pretensiones de dominio. La centralidad del personaje que éste mismo intentaba imponer, a costa de la cohesión entre su vida íntima y los eventos externos, puede finalmente fluir en favor de una centralidad de la trama que concede espacio a los pensamientos del personaje en armonía con ella.

Cabe ahora preguntarnos si los propósitos de autonomía de la autora permiten al menos suponer alguna especie de valoración ética suya sobre los actos del protagonista: ¿qué tan lícito es imaginar un sentido otorgado a las dificultades de Jake para convivir con su existencia? ¿Qué valor tiene el conflicto entre su determinación y los acontecimientos adversos que reconfiguran su plan de vida $\mathrm{y}$, al mismo tiempo, su camino de aprendizaje, inicialmente contrario a los eventos que le esperan? Sobre la existencia y la posibilidad de revisar un juicio moral en Bajo la red, encontramos un interesante comentario en el análisis que la misma Murdoch hace con respecto a una pregunta - lo que el autor revela sobre sus presuposiciones morales - en la entrevista citada con Bryan Magee. Al final de una elaborada respuesta, en la que recuerda que literatura y filosofía son tanto la producción de sujetos 
autónomos como el reflejo de un mundo, afirma que un escritor "tiene que juzgar su personaje y tomar parte [...] con la ambigüedad y la mistificación que entran en el asunto, [...] jugar todo tipo de juego mistificador con la manera en que se quiere revelar su personaje [...]. La revelación está ahí y el juicio está ahí" [la traducción es mía] (Murdoch, 1978).

\section{Conclusiones}

En el contexto del citado juego mistificador, se ha podido observar cómo las meditaciones de Paul Ricoeur sobre las dimensiones fragmentarias del sí mismo, la necesaria otredad en la que éstas se configuran y el papel revelador de la dimensión narrativa, no sólo son recurrentes en la filosofía contemporánea, sino que constituyen elementos de los cuales una autora como Iris Murdoch puede ser consiente y decidir integrarlos en una novela, a partir de su peculiar posición de configuradora de ficción con temas derivados de la reflexión filosófica.

Los razonamientos ricoeurianos sobre la naturaleza tríplice de identidad idem, ipse, y el reconocimiento al individuo de y por la otredad, permiten ampliar el alcance del análisis literario hacia una mejor definición de los modos de presentación, límites y evolución del personaje. Del mismo modo, la interesante transformación de la perspectiva en la narración de Bajo la red constituye un campo de aplicación particularmente significativo. Lo anterior permite explorar las formas en que se expresa la consciencia de sí en esta novela, en la narración en primera persona de Jake, en su soliloquio y en las voces de los otros que aparecen, sobre todo, en la narración a menudo atemporal — casi encerrada en la ciclicidad de un relato picaresco - de la primera parte de la obra. Igualmente, se puede apreciar su desarrollo - en una especie de Bildungsroman o novela de aprendizaje existencial- hacia una consideración más completa de su ser y su apertura en relación con lo externo a él, los otros personajes y el mundo que sus vivencias le han enseñado a entender mejor. De esta manera, la obra llega a reafirmar no sólo la libertad de pensamiento y acción que la autora siente que les debe dar a sus personajes, sino un crecimiento de la personalidad de dichas identidades narrativas y una primacía de la importancia de este mismo desarrollo sobre los acontecimientos de la trama. En novelas como Bajo la red, entonces, la evolución del personaje constituye el verdadero tema de la trama y no un elemento prescindible de una acción que la expresa. Resulta evidente, y tal vez más reconocible aún, gracias al presente análisis, una visión integrada de la propiedad que Kermode atribuye a la ficción en el ya citado concepto 'sentido de un final': un sentido general del cuento de sí que Jake desarrolla en primera persona, y que reconoce en más de un momento de comentario metaficcional de su narración (por ejemplo, 21 y 250).

Queda abierta la invitación a un estudio de mayor extensión, a partir del modelo aquí presentado, que incluya un análisis similar de una agrupación del género novelesco o, tal vez, de la producción de Iris Murdoch: ${ }^{7}$ en particular, en este segundo caso, podrían ser considerados sus escritos filosóficos ligados a la percepción intersubjetiva y al examen de la dimensión sensorial y de la sensibilidad, en conexión con los temas de la otredad moral desarrollados en los capítulos 7 y 8 de Oneself as another, de Ricoeur.

7 Resumida por Martha Nussbaum en su ensayo "When she was good" (2001: 28-34). 


\section{REFERENCIAS}

James, Henry (1934), The art of the novel, Chicago, The University of Chicago Press.

Kermode, Frank (1978), The Genesis of Secrecy. On the Interpretation of Narrative, Cambridge, Harvard University Press.

Murdoch, Iris (1954), Under the Net, Londres, Penguin Books.

Murdoch, Iris (24 de mayo de 1965), Modern Novelists - Interview with Iris Murdoch, entrevista por Frank Kermode, Rodney Bennett (prod.), For Sixth Forms: Modern Novelists: 3: Iris Murdoch, Author of An Unofficial Rose [transmisión televisiva], Londres, BBC, disponible en: https://youtu.be/annRHY8Gcdw

Murdoch, Iris (20 de abril de 1978), Philosophy and Literature with Iris Murdoch, entrevista por Bryan Magee, Janet Hoenig (prod), Men of Ideas [transmisión televisiva], Londres, BBC, disponible en: https://youtu.be/pBG10XnxQaI

Nussbaum, Martha C. (2001), "When she was good", en The New Republic, Washington, 31 de diciembre de 2001, pp. 28-34.

Ricoeur, Paul (1966), Freedom and Nature. The Voluntary and the Involuntary, vol. 1, Evanston, Northwestern University Press.

Ricoeur, Paul (1985), Tiempo y narración, México, Siglo XXI Editores.

Ricoeur, Paul (1990), Oneself as another, Chicago, The University of Chicago Press.

Andrea Coghi. Maestro en Lengua y Literaturas Extranjeras por la Università degli Studi di Milano, Italia. Estudiante del Doctorado en Ciencias del Lenguaje por la Benemérita Universidad Autónoma de Puebla (BUAP), México. Entre sus intereses académicos se encuentran la teoría de la literatura aplicada al aprendizaje de idiomas extranjeros, al análisis literario y a la transposición cinematográfica; así como las tecnologías digitales para la docencia. Entre sus últimas publicaciones se encuentra: "Soldados y marinos en las novelas en inglés: de la construcción de una identidad personal a la exploración ética" (AEH. Revista de Artes y Humanidades, núm. 10). 


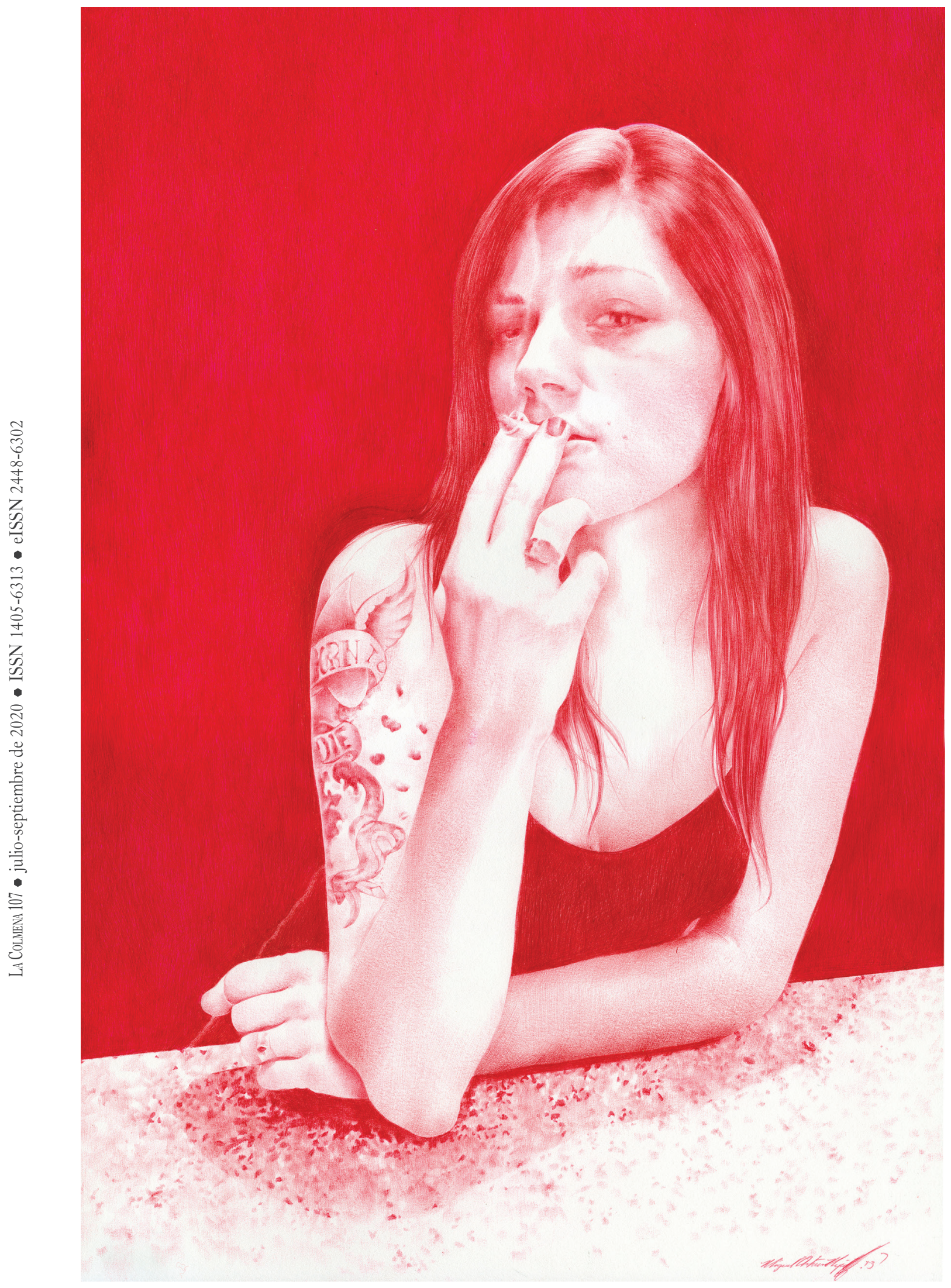

La impertinencia del silencio de la serie [En]soñaciones (2013). Bolígrafo sobre papel: Miguel Arturo Mejía-Martínez. Prohibida su reproducción en obras derivadas. 